\section{OPEN ACCESS}

Edited by: Christoph Anacker,

Columbia University Irving Medical

Center, United States

Reviewed by:

Paul J. Lucassen,

University of Amsterdam, Netherlands

Yoshihiko Matsumoto,

Yamagata University, Japan

*Correspondence:

Naguib Mechawar

naguib.mechawar@mcgill.ca

Specialty section: This article was submitted to

Molecular Psychiatry

a section of the journal

Frontiers in Psychiatry

Received: 12 December 2020 Accepted: 15 January 2021 Published: 04 February 2021

Citation:

O'Leary LA, Belliveau C, Davoli MA,

Ma JC, Tanti A, Turecki G and

Mechawar N (2021) Widespread

Decrease of Cerebral

Vimentin-Immunoreactive Astrocytes

in Depressed Suicides.

Front. Psychiatry 12:640963.

doi: 10.3389/fpsyt.2021.640963

\title{
Widespread Decrease of Cerebral Vimentin-Immunoreactive Astrocytes in Depressed Suicides
}

\begin{abstract}
Liam Anuj O'Leary ${ }^{1,2}$, Claudia Belliveau ${ }^{1,2}$, Maria Antonietta Davoli ${ }^{1}$, Jie Christopher Ma ${ }^{1}$, Arnaud Tanti ${ }^{1}$, Gustavo Turecki ${ }^{1,2,3}$ and Naguib Mechawar ${ }^{1,2,3 *}$

${ }^{1}$ McGill Group for Suicide Studies, Douglas Mental Health University Institute, Verdun, QC, Canada, ${ }^{2}$ Integrated Program in Neuroscience, McGill University, Montreal, QC, Canada, ${ }^{3}$ Department of Psychiatry, McGill University, Montreal, QC, Canada
\end{abstract}

Post-mortem investigations have implicated cerebral astrocytes immunoreactive (-IR) for glial fibrillary acidic protein (GFAP) in the etiopathology of depression and suicide. However, it remains unclear whether astrocytic subpopulations IR for other astrocytic markers are similarly affected. Astrocytes IR to vimentin (VIM) display different regional densities than GFAP-IR astrocytes in the healthy brain, and so may be differently altered in depression and suicide. To investigate this, we compared the densities of GFAP-IR astrocytes and VIM-IR astrocytes in post-mortem brain samples from depressed suicides and matched non-psychiatric controls in three brain regions (dorsomedial prefrontal cortex, dorsal caudate nucleus and mediodorsal thalamus). A quantitative comparison of the fine morphology of VIM-IR astrocytes was also performed in the same regions and subjects. Finally, given the close association between astrocytes and blood vessels, we also assessed densities of CD31-IR blood vessels. Like for GFAP-IR astrocytes, VIM-IR astrocyte densities were found to be globally reduced in depressed suicides relative to controls. By contrast, CD31-IR blood vessel density and VIM-IR astrocyte morphometric features in these regions were similar between groups, except in prefrontal white matter, in which vascularization was increased and astrocytes displayed fewer primary processes. By revealing a widespread reduction of cerebral VIM-IR astrocytes in cases vs. controls, these findings further implicate astrocytic dysfunctions in depression and suicide.

Keywords: human, post-mortem, depression, suicide, astrocyte, vimentin, GFAP

\section{INTRODUCTION}

Astrocytes were first identified as a glial cell type in the human brain more than a 100 years ago, and until a few decades ago were mostly seen to have a passive role of providing nutritional support for neurons (1). Animal studies have since revealed that astrocytes can strongly modulate most facets of neuronal activity, including neuronal firing, neurotransmitter synthesis, neurotransmitter reuptake, and synaptic transmission (2-6). Astrocytes might especially influence neuronal activity in the human brain, as they are almost three-fold larger in volume and four-fold faster at signaling in the human cortex than in the mouse cortex $(7,8)$.

The first post-mortem investigations of major depressive disorder (MDD) reported reduced glial (but not neuronal) densities in the ventral anterior cingulate cortex (9), the orbitofrontal cortex (10), and the amygdala (11). These findings were later attributed to a reduced number 
of cerebral astrocytes, particularly those immunoreactive (-IR) for the astrocyte-specific marker glial fibrillary acidic protein (GFAP). Post-mortem brain samples from depressed individuals have fewer GFAP-IR astrocytes (12-17), and lower levels of GFAP mRNA and protein (18-20). Of all psychiatric conditions, GFAP is most implicated in MDD (21). However, GFAP labels only a minority of astrocytes, and so may misrepresent astrocytic phenotypes in MDD (22). For instance, the hippocampal CA1 region has lower densities of S100B-IR, but not GFAP-IR, astrocytes in MDD (23). Conversely, the amygdala has lower densities of GFAP-IR, but not S100B-IR, astrocytes in MDD (15, 24). Hence, GFAP expression might not capture the widespread phenotype of astrocyte dysfunction in post-mortem studies of MDD, including morphometric differences in cortical fibrous astrocytes (25), reduced vascular coverage (26), differential methylation patterns for genes enriched in astrocytes (27), and abnormally low expression levels of astrocytic glutamate transporters $(18,27,28)$, and gap junction proteins (29-31).

Like GFAP, vimentin (VIM) is a type III intermediate filament that is strongly expressed in cerebral astrocytes, however, it is also expressed in vascular endothelial cells. IR astrocytes has been relatively rare, especially given the functional relationship between VIM and GFAP proteins. For instance, VIM has a reciprocal expression profile with GFAP during development, can functionally compensate for the transgenic loss of GFAP expression, and is peculiarly absent in Rosenthal fibers-a defining pathological feature of Alexander disease, a genetic condition associated with GFAP mutations (32-34). A previous post-mortem study has found qualitative differences in VIMIR astrocytes in neurological conditions (35). In a recent postmortem study, we characterized VIM immunoreactivity in different cortical and subcortical brain regions using samples from healthy individuals having died suddenly, and found that VIM-IR astrocytes had different densities from GFAP-IR astrocytes, but that both GFAP-IR and VIM-IR astrocyte density inversely correlated with CD31-IR vascular density (8). We were then interested in how these findings might relate to depression.

Here, we compare the densities of GFAP-IR astrocytes, VIMIR astrocytes and CD31-IR blood vessels in three brain regions from depressed suicides and matched non-psychiatric controls. In addition, we assessed VIM-IR astrocyte morphometry in MDD in the same sections. In depressed suicides, we found a general and consistent reduction in the density of GFAP-IR and VIM-IR astrocytes, as well as a significant increase in CD31-IR vascularization in the prefrontal cortex white matter. However, we observed almost unaltered VIM-IR astrocyte morphometry in depressed suicides relative to controls. These findings indicate that in the brains of depressed individuals, regional variations in astrocyte densities are much stronger and widespread than changes in astrocyte morphometry.

\section{MATERIALS AND METHODS}

\section{Subjects and Tissue Processing}

This study was approved by the Douglas Hospital Research Ethics Board. Brain samples were analyzed from adult Caucasian male depressed suicides $(n=10)$ and non-psychiatric controls $(n=$
TABLE 1 | Subject information.

\begin{tabular}{lcc}
\hline & $\begin{array}{c}\text { Controls }(\boldsymbol{n}=\mathbf{1 0}) \\
\text { Mean } \pm \text { SEM }\end{array}$ & $\begin{array}{c}\text { Depressed } \\
\text { suicides }(\boldsymbol{n}=\mathbf{1 0}) \\
\text { Mean } \pm \text { SEM }\end{array}$ \\
\hline Age & $40.6 \pm 5.0$ & $38.5 \pm 4.2$ \\
Sex & $10 \mathrm{M}$ & $10 \mathrm{M}$ \\
Tissue pH & $6.5 \pm 0.0$ & $6.6 \pm 0.0$ \\
PMl (h) & $15.3 \pm 2.8$ & $21.8 \pm 5.9$ \\
Cause of death & 8 cardiovascular, 2 & 10 hanging \\
& accidental falls & 10 \\
Depression status & 0 & 3 \\
Toxicology report & & 2 \\
Alcohol & 1 & 1 \\
Cocaine & 0 & (desvenlafaxine) \\
Antidepressants & 0 & \\
\end{tabular}

TABLE 2 | Antibodies: specifications.

\begin{tabular}{lllll}
\hline Primary antibody & Species & Clone & Dilution & Source \\
\hline Vimentin & Rabbit & ab92547 & $1: 500$ & Abcam \\
GFAP & Chicken & ab4674 & $1: 1,000$ & Abcam \\
CD31 & Mouse & JC70 & $1: 250$ & Santa Cruz \\
\hline
\end{tabular}

10). Subject information is available in Table 1. All depressed suicides died during a major depressive episode. All controls died suddenly without any known inflammatory, psychiatric or neurological disorder. Brain donation and psychiatric diagnosis were as described previously (25). Prior to tissue selection, optimal subject groups were matched for three covariates, which were assessed using paired $t$-tests: age $(p=0.75)$, tissue $\mathrm{pH}$ $(p=0.40)$, and post-mortem interval (PMI; $p=0.38)$. For all 20 subjects, three brain regions were dissected from thick frozen sections: the dorsomedial prefrontal cortex [Brodmann Area (BA) 8/9], the dorsal caudate nucleus (precommissural) and the mediodorsal thalamus. These brain regions were selected as our recent study characterized GFAP-IR and VIM-IR astrocytes in healthy adults in these regions, and previous work from our group and collaborators suggested gene expression differences in astrocytes of these regions in depression $(8,20,27)$. We studied the PFC gray matter (PFC GM) and white matter (PFC WM) independently. Fresh-frozen $1 \mathrm{~cm}^{3}$ tissue blocks from each region were fixed overnight in $10 \%$ formalin, suspended in $30 \%$ sucrose solution until equilibrium was reached, flash-frozen in $-35^{\circ} \mathrm{C}$ isopentane, and cut on a sliding microtome into $50 \mu \mathrm{m}$-thick serial sections that were stored at $-20^{\circ} \mathrm{C}$ in a cryoprotectant solution until processing for immunohistochemistry (IHC). Immunolabeling involved independently using each antibody listed in Table 2 within a conventional DAB IHC protocol and a separate stereological series of sections, as described previously (8).

\section{Stereology and Morphology}

Stereological cell counting and live tracing was performed as described previously (8), while additionally blinded to subject 
identities. Reliable stereological estimates, with a Gunderson coefficient of error (CE, $m=1)<0.10$, were obtained by sampling the following percentage areas from section contours: $5 \%$ for GFAP in all regions; and 10\% for VIM in the PFC GM and the caudate nucleus, $25 \%$ in the PFC WM, and $100 \%$ in the mediodorsal thalamus. A total of 320 VIM-IR astrocytes were reconstructed, four from the PFC GM, the PFC WM, the thalamus and the caudate nucleus of each subject.

\section{Vascular Density}

To estimate vascular density for its comparison with stereological cell densities, 15 brightfield images of CD31-IR vasculature were taken across four sections of a stereological series of sections for each region at low (10X objective) magnification. Using ImageJ software (NIH, USA), each image was converted into an 8-bit format and then manually thresholded to remove most background staining before the percentage area was measured. To reduce noise from background staining and artifacts, only 10 values closest to the original mean value were used to calculate the mean \% CD31-IR density for each region and subject.

\section{Morphometric Features}

With the same slides and workstation used for stereological analysis, the morphometry of VIM-IR astrocytes were manually traced live using a 100X oil immersion objective and a computerbased tracing system (Neurolucida Explorer, MBF Bioscience). Immunostained cells were randomly selected, but had to display the following features in ordered to be selected for reconstruction: (1) unobstructed by neighboring cells; (2) of representative size and shape; (3) of equal staining across cellular compartments; (4) contained within the thickness of the section; (5) forming clear endfeet contacts with a VIM-IR blood vessel. A total of 320 VIM-IR cells were traced in this study, as four cells per region were reconstructed and analyzed for all three regions of all 20 subjects (where cortical gray and white matter were considered as independent compartments). Analyses were performed on all reconstructed cells with Neurolucida Explorer (MBF Bioscience) by an experimenter blinded to the group identity of each sample. Branched Structure Analysis (BSA) was used to compare seven structural features of astrocytes: process number, node number, terminal number, mean process length, total process length, mean process area, and soma area.

\section{D Reconstructions}

All 3D reconstructions were created in Blender (Amsterdam, the Netherlands), which is a free, open-source 3D software suite. For the morphological reconstructions featured in Figure 3A, Neurolucida .DAT files were converted online into .SWC files for two morphological tracings of VIM-IR astrocytes in the prefrontal cortex white matter, which had process numbers representative of controls and depressed suicides in this region. These were then imported into Blender with the assistance of the Neuromorphovis plugin, and BSA features were manually colored and annotated for demonstration purposes (36). For the "stereological cube" models featured in Figure 5, a randomly distributed particle system of $25 \mathrm{~mm}^{3}$ spheres within the volume of $1 \mathrm{~m}^{3}$ cube was used to visualize regional densities of $25 \mu \mathrm{m}^{3}$ diameter cell bodies counted within the regional brain volume of $1 \mathrm{~mm}^{3}$. Sphere volume was kept constant across markers and regions to facilitate cell density comparisons.

\section{Statistical Analysis}

All measurements are expressed as mean \pm standard error of the mean (SEM), graphs display corrected $p$-values on data after outlier removal, and $p<0.05$ was considered significant in all statistical tests. Preliminary statistical analyses were performed using Prism v. 6.04 (GraphPad Software, San Diego, CA, USA). Data were assessed for a normal distribution using the Kolmogorov-Smirnov-test. Statistical outliers were identified using a ROUT outlier test with $Q=1 \%$, which is a conventionally used method for identifying outliers (37). We decided to remove outliers rather than perform non-parametric tests as very few outliers were present in this study and it facilitated more meaningful comparisons by allowing the same statistical tests to be used for all related data sets. Three data points were identified as outliers in this study, one for VIM-IR astrocyte density in PFC GM of a depressed suicide, and two for GFAPIR astrocyte density in the thalamus of depressed suicides. In these two instances we provide in-text the $p$-value before outlier removal, as assessed by the Mann-Whitney-test-outliers were removed to normalize both data sets, so they met parametric test assumptions. All other instances of uncorrected and corrected group differences were assessed for each region using unpaired $t$-tests using SPSS version 21 software (IBM Corporation), correction was made for three covariates-age, $\mathrm{pH}$ and $\mathrm{PMI}-$ to correct for any potential effects they may have had on density or morphometry measurements.

\section{RESULTS}

\section{GFAP-IR Astrocyte Densities Are Generally Reduced in Depressed Suicides}

Using a stereological approach to assess the regional densities of astrocytes in an unbiased fashion, GFAP-IR astrocytes were found to be lower in all regions for depressed suicides relative to controls (Figure 1A). Prefrontal cortex gray matter had half as many GFAP-IR astrocytes in depressed suicides than in controls $\left[444 \pm 73\right.$ cells $/ \mathrm{mm}^{3}$ vs. $1,035 \pm 232$ cells $/ \mathrm{mm}^{3} ; p$ $($ uncorrected $)=0.03, p($ corrected $)=0.04]$. Prefrontal cortex white mater had almost half as many GFAP-IR astrocytes in depressed suicides than in controls, although this difference was not statistically significant $\left[1,353 \pm 373\right.$ cells $/ \mathrm{mm}^{3}$ vs. 2,668 \pm 510 cells $/ \mathrm{mm}^{3} ; p$ (uncorrected $)=0.05, p$ (corrected $)=$ 0.07]. The mediodorsal thalamus had the greatest difference between groups, with depressed suicides displaying 5-fold fewer GFAP-IR astrocytes than depressed suicides $[1,049 \pm 400$ cells $/ \mathrm{mm}^{3}$ vs. $5,902 \pm 1,348$ cells $/ \mathrm{mm}^{3} ; p$ (uncorrected) $=$ 0.003 ], but this became 12 -fold after removing one statistical outlier that prevented parametric testing $\left[491 \pm 101 \mathrm{cells} / \mathrm{mm}^{3}\right.$ vs. $5,902 \pm 1,348$ cells $/ \mathrm{mm}^{3} ; p$ (uncorrected) $=0.003, p$ $($ corrected $)=0.005]$. Similarly, the caudate nucleus also had 5 -fold fewer GFAP-IR astrocytes in depressed suicides than in controls $\left[204 \pm 87\right.$ cells $/ \mathrm{mm}^{3}$ vs. $1,142 \pm 356$ cells $/ \mathrm{mm}^{3} ; p$ $($ uncorrected $)=0.02, p($ corrected $)=0.03$ ] 


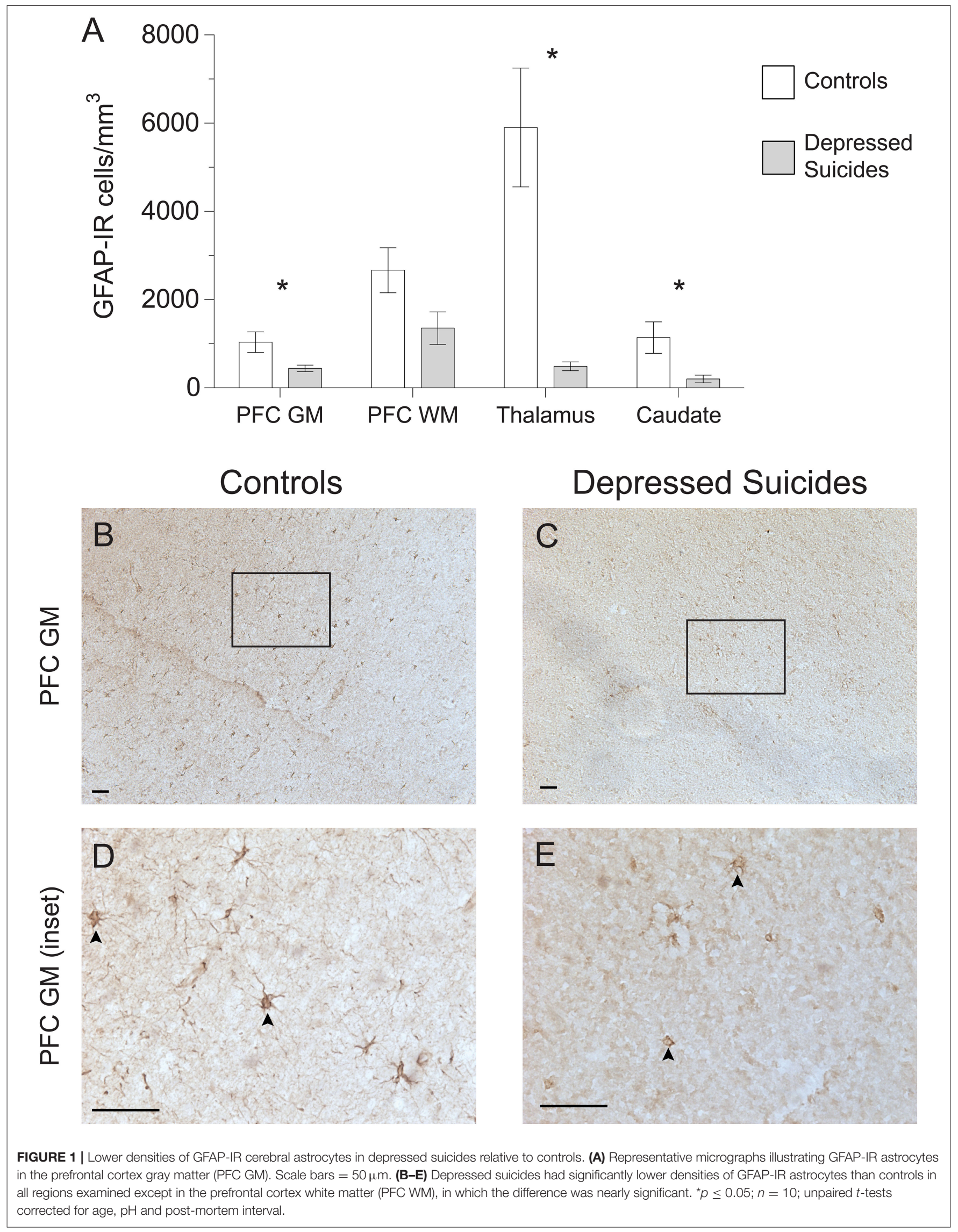




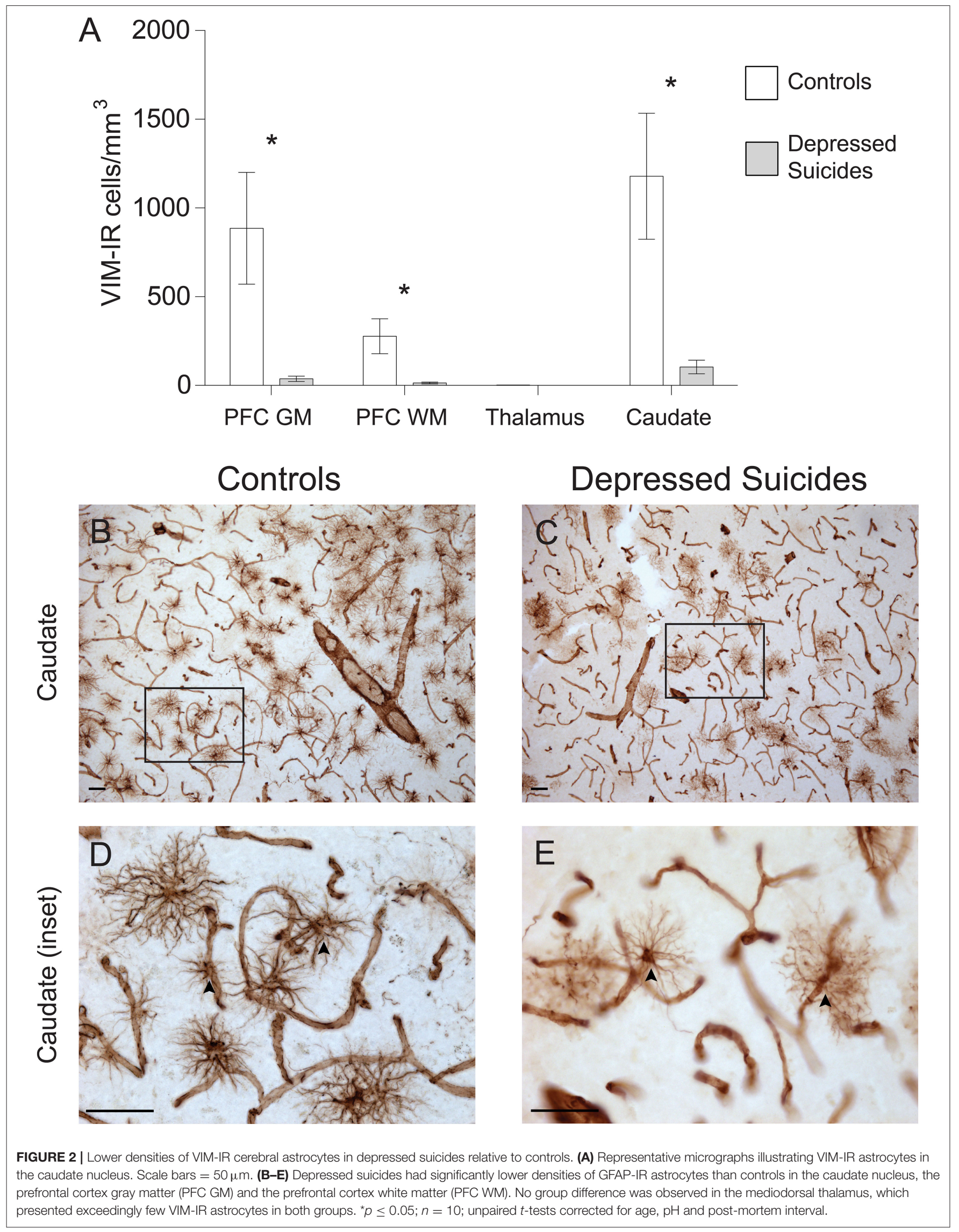




\section{Vimentin-IR Astrocyte Densities Are Generally Reduced in Depressed Suicides}

As the regional densities of GFAP-IR and VIM-IR were recently reported to differ in post-mortem samples from healthy human brains (8), we next assessed whether VIM-IR astrocytes would also display altered regional densities in depressed suicides (Figure 2A). Prefrontal cortex gray matter had 6-fold fewer VIMIR astrocytes in depressed suicides than in controls $[137 \pm 100$ vs. $886 \pm 316$ cells $/ \mathrm{mm}^{3} ; p$ (uncorrected) $\left.=0.08\right]$, and this difference became over 20 -fold and statistically significant after removing one statistical outlier [ $38 \pm 15 \mathrm{vs.} 886 \pm 316 \mathrm{cells} / \mathrm{mm}^{3}$; $p($ uncorrected $)=0.02, p$ (corrected) $=0.03$ ]. Prefrontal cortex white matter had 20-fold fewer VIM-IR astrocytes in depressed suicides than in controls $[14 \pm 5$ vs. $278 \pm 98$ cells $/ \mathrm{mm}^{3} ; p$ (uncorrected) $=0.002, p$ (corrected) $\left.=0.001\right]$. There were slightly fewer VIM-IR astrocytes in the mediodorsal thalamus of controls relative to depressed suicides, but this was not statistically significant due to there being very few cells in both groups $\left[1.2 \pm 0.2\right.$ vs. $2.1 \pm 0.5$ cells $/ \mathrm{mm}^{3} ; p$ $($ uncorrected $)=0.12, p($ corrected $)=0.05]$. The caudate nucleus had 10-fold fewer VIM-IR astrocytes in depressed suicides than in controls $\left[104 \pm 38\right.$ vs. $1,179 \pm 355$ cells $/ \mathrm{mm}^{3} ; p$ $($ uncorrected $)=0.01, p($ corrected $)=0.01]$.

\section{Vimentin-Immunoreactive Astrocytes: Mostly Unchanged Morphology in Depressed Suicides}

\section{Process Structure}

The morphology of VIM-IR astrocytes mostly did not differ significantly between depressed suicides and controls (Figure 3; see parameters assessed in $\mathbf{3 A}$ ). The only statistically significant group difference for process number was in the prefrontal cortex white matter (Figure 3B); relative to controls, astrocytes from depressed suicides had on average two more processes in prefrontal cortex gray matter [ $30 \pm 1$ vs. $28 \pm 2$ processes; $p$ $($ uncorrected $)=0.60, p$ (corrected $)=0.83$ ], six fewer processes in prefrontal white matter $[29 \pm 2$ vs. $35 \pm 1$ processes; $p($ uncorrected $)=0.008, p($ corrected $)=0.02]$, three fewer processes in the thalamus [18 \pm 1 vs. $21 \pm 1$ processes; $p$ (uncorrected) $=0.07, p$ (corrected) $=0.06]$, and the same number of processes in the caudate nucleus $[27 \pm 1$ vs. 27 \pm 1 processes; $p$ (uncorrected) $=0.63, p$ (corrected) $=0.77$ ] Astrocyte node number did not significantly differ between groups for any region (Figure 3C); relative to controls, astrocytes from depressed suicides had on average three fewer nodes in prefrontal cortex gray matter $[30 \pm 3$ vs. $33 \pm 2$ nodes; $p$ $($ uncorrected $)=0.47, p($ corrected $)=0.26]$, two fewer nodes in prefrontal white matter $[20 \pm 2$ vs. $22 \pm 2$ nodes; $p$ (uncorrected) $=0.50, p($ corrected $)=0.59$ ], the same number of nodes in the thalamus [ $14 \pm 1$ vs. $14 \pm 2$ nodes; $p$ (uncorrected) $=0.85, p$ (corrected) $=0.95]$, and one less node in the caudate nucleus [ 32 \pm 3 vs. $33 \pm 3$ nodes; $p$ (uncorrected $)=0.82, p($ corrected $)=$ 0.91]. Similarly, terminal number was not significantly affected in depressed suicides (Figure 3D); relative to controls, astrocytes from depressed suicides had on average two fewer terminals in prefrontal cortex gray matter $[60 \pm 4$ vs. $62 \pm 4$ terminals; $p$
$($ uncorrected $)=0.70, p($ corrected $)=0.43$ ] , eight fewer terminals in prefrontal white matter $[49 \pm 2$ vs. $57 \pm 3$ terminals; $p$ $($ uncorrected $)=0.05, p($ corrected $)=0.08]$, two fewer terminals in the thalamus $[33 \pm 2$ vs. $35 \pm 2$ terminals; $p$ (uncorrected) $=$ $0.39, p$ (corrected) $=0.33$ ], and the same number of terminals in the caudate nucleus $[61 \pm 3$ vs. $61 \pm 3$ terminals; $p$ (uncorrected) $=0.94, p($ corrected $)=0.87]$.

\section{Process Length}

Astrocyte total process length did not significantly differ between groups in any region (Figure 3E); relative to controls, the mean total process length for astrocytes from depressed suicides was consistently lower in the prefrontal cortex gray matter $[1,800 \pm$ 108 vs. $1,911 \pm 108 \mu \mathrm{m} ; p$ (uncorrected) $=0.47, p$ (corrected) $=0.29]$, the prefrontal white matter $[1,750 \pm 98$ vs. $1,968 \pm$ $133 \mu \mathrm{m} ; p$ (uncorrected) $=0.21, p$ (corrected) $=0.29]$, the thalamus $[1,100 \pm 121$ vs. $1,192 \pm 92 \mu \mathrm{m} ; p$ (uncorrected) $=$ $0.55, p($ corrected $)=0.46]$, and the caudate nucleus $[1,798 \pm$ 91 vs. $1,882 \pm 126 \mu \mathrm{m} ; p($ uncorrected $)=0.59, p($ corrected $)=$ $0.79]$. Across all regions astrocyte mean process length did not significantly or consistently differ between groups (Figure 3F); relative to healthy controls, astrocyte processes from depressed suicides were shorter in prefrontal cortex gray matter $[62 \pm 3 \mathrm{vs.}$ $68 \pm 2 \mu \mathrm{m} ; p$ (uncorrected $)=0.14, p$ (corrected $)=0.14]$, longer in prefrontal white matter $[62 \pm 1$ vs. $57 \pm 2 \mu \mathrm{m} ; p$ (uncorrected) $=0.06, p$ (corrected) $=0.08]$, longer in the thalamus $[62 \pm 5$ vs. $58 \pm 4 \mu \mathrm{m} ; p$ (uncorrected $)=0.56, p$ (corrected) $=0.73$ ] and shorter in the caudate nucleus $[67 \pm 4$ vs. $71 \pm 4 \mu \mathrm{m} ; p$ $($ uncorrected $)=0.39, p($ corrected $)=0.53$.

\section{Cell Size}

The surface area of astrocyte processes did not significantly differ between groups for any region (Figure 3G); relative to controls, the surface area of astrocyte processes from depressed suicides was smaller in the prefrontal cortex gray matter $[1,377 \pm 79$ vs. $1,401 \pm 112{\mu \mathrm{m}^{2}}^{2} p$ (uncorrected) $=0.86, p$ (corrected) $=0.80]$, the prefrontal white matter $[1,381 \pm 119$ vs. $1,625 \pm$ $119 \mu \mathrm{m}^{2} ; p$ (uncorrected) $=0.16, p$ (corrected) $=0.20$ ], the thalamus $\left[907 \pm 133\right.$ vs. $1,019 \pm 98 \mu \mathrm{m}^{2} ; p=$ (uncorrected) $0.50, p($ corrected $)=0.47]$, and the caudate nucleus $[1,519 \pm$ 81 vs. $1,657 \pm 128 \mu \mathrm{m}^{2} ; p$ (uncorrected) $=0.38, p$ (corrected) $=0.53]$. As for total process length, the volume occupied by astrocyte processes did not significantly differ between group in any region (Figure $3 \mathbf{H}$ ), but relative to healthy controls, the mean volume of astrocyte processes from depressed suicides was consistently smaller in the prefrontal cortex gray matter $[87 \pm$

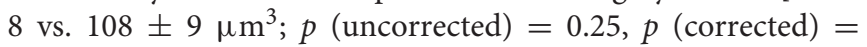
$0.24]$, the prefrontal white matter $\left[102 \pm 12 \mathrm{vs} .132 \pm 14 \mu \mathrm{m}^{3}\right.$; $p($ uncorrected $)=0.30, p($ corrected $)=0.16]$, the thalamus $[63$ \pm 10 vs. $89 \pm 12 \mu \mathrm{m}^{3} ; p$ (uncorrected) $=0.31, p$ (corrected) $=0.30]$, and the caudate nucleus $\left[121 \pm 8\right.$ vs. $140 \pm 14 \mu \mathrm{m}^{3}$; $p($ uncorrected $)=0.32, p($ corrected $)=0.25]$. The soma area of astrocytes did not significantly differ between groups for any region (Figure 3I); relative to healthy controls, the soma area of astrocytes from depressed suicides was smaller in the prefrontal cortex gray matter $\left[69 \pm 5\right.$ vs. $70 \pm 5 \mu \mathrm{m}^{2} ; p$ (uncorrected) $=$ $0.92, p$ (corrected $)=0.76]$, smaller in the prefrontal white matter 


\section{A}
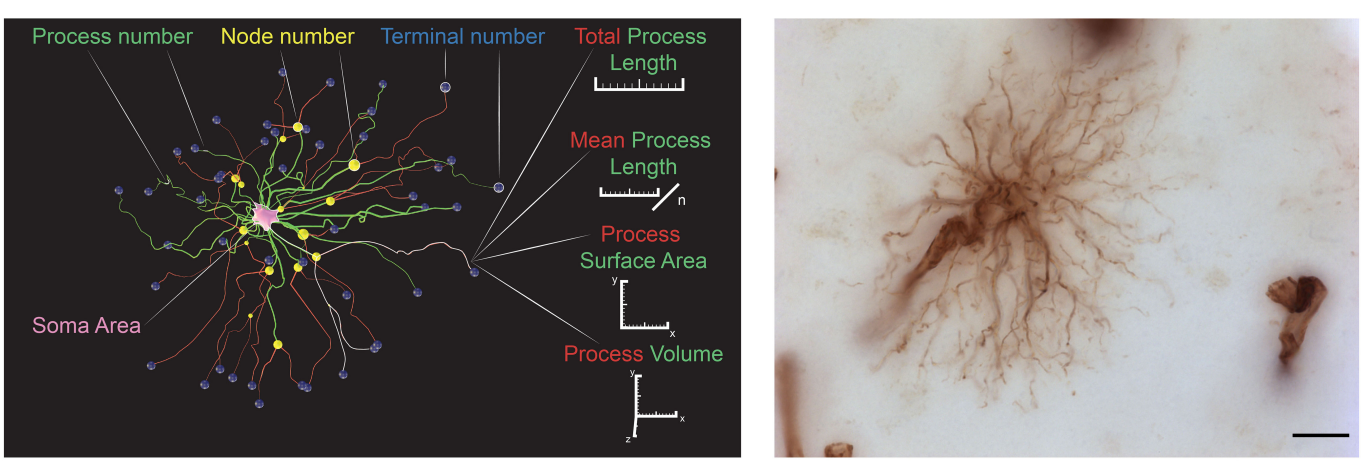

B

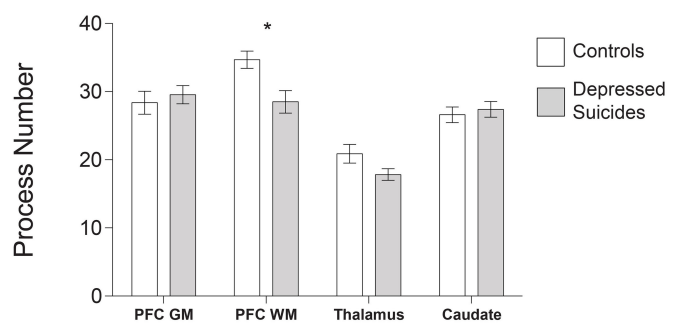

D

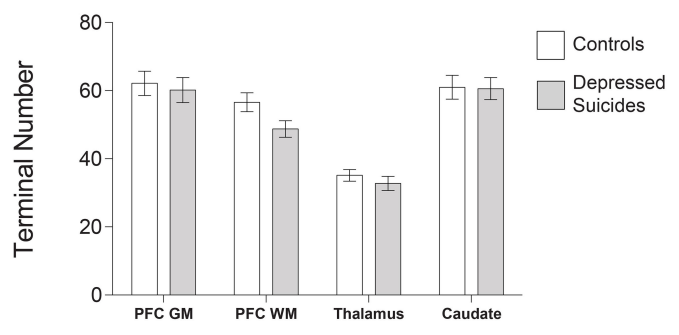

$\mathrm{F}$

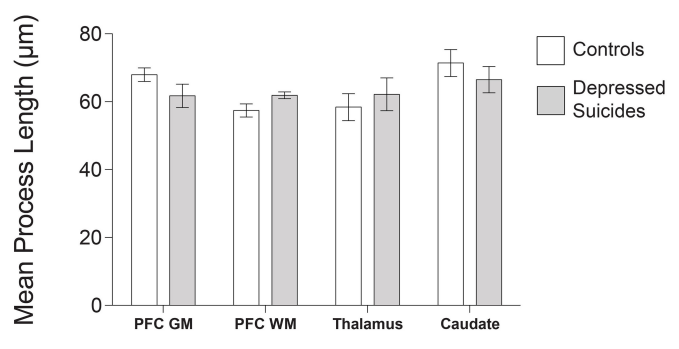

$\mathrm{H}$

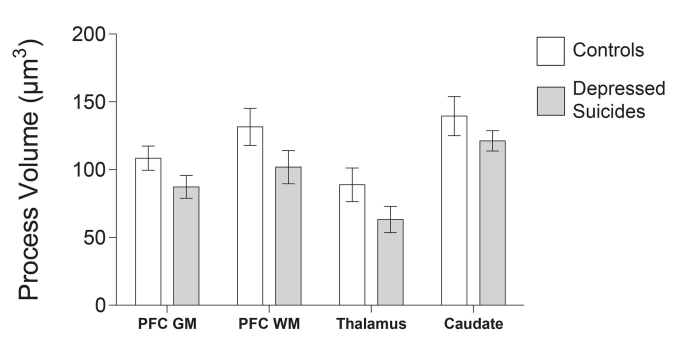

C

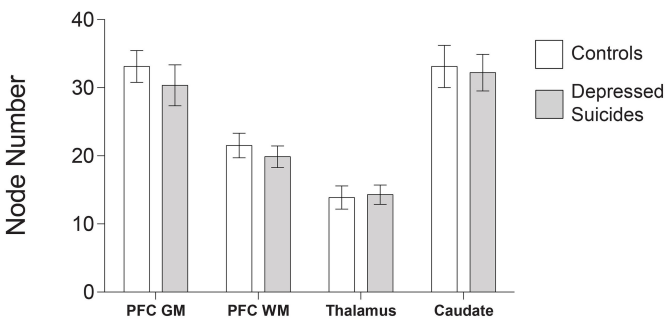

E

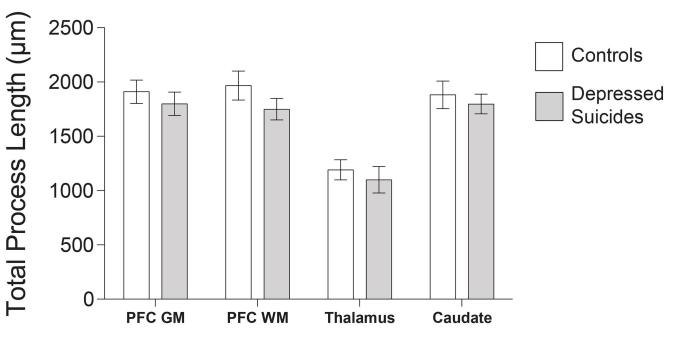

G

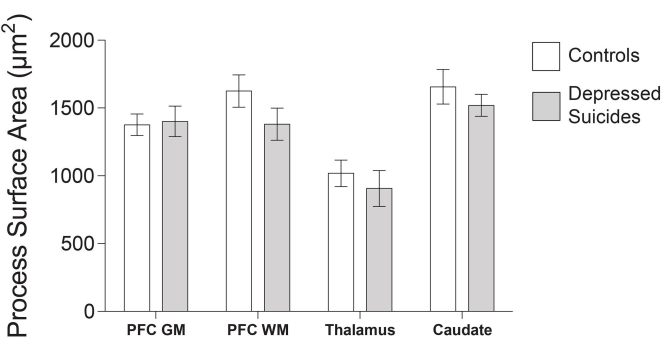

I

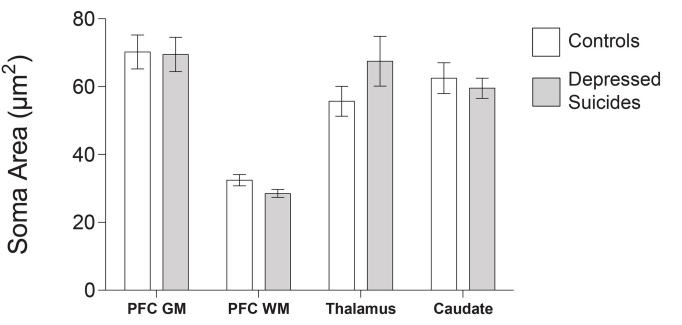

FIGURE 3 | VIM-IR astrocyte morphology is generally similar in depressed suicides vs. controls. (A) 3D reconstruction of a VIM-IR astrocyte from the prefrontal cortex white matter (PFC WM) representative of those from depressed suicides (left). The soma (pink) that extends primary processes (green) which can branch at nodes (yellow) into secondary processes (red) that eventually end as terminals (blue). Branched Structure Analysis (BSA) measurements have been annotated (left), and a minimum intensity projection of a representative VIM-IR astrocyte from the caudate nucleus of a depressed suicide is illustrated (right). (B-I) The BSA revealed a lower (primary) process number for VIM-IR astrocytes in the PFC WM of depressed suicides relative to controls. There were no group differences for any BSA

measurements of VIM-IR astrocytes in the prefrontal cortex gray matter (PFC GM), thalamus or caudate nucleus. ${ }^{\star} p \leq 0.05 ; n=10$; unpaired $t$-tests corrected for age, $\mathrm{pH}$ and post-mortem interval. 
[29 \pm 1 vs. $32 \pm 2 \mu \mathrm{m}^{2} ; p$ (uncorrected) $=0.07, p$ (corrected) $=0.06]$, larger in the thalamus [68 \pm 7 vs. $56 \pm 4 \mu \mathrm{m}^{2} ; p$ $($ uncorrected $)=0.19, p($ corrected $)=0.24]$, and smaller in the caudate nucleus $\left[60 \pm 3\right.$ vs. $62 \pm 5 \mu \mathrm{m}^{2} ; p$ (uncorrected) $=0.60$, $p($ corrected $)=0.50]$.

\section{CD31-Immunoreactive Vascular Density Is Increased in the Prefrontal Cortex White Matter of Depressed Suicides}

There was no general trend for group differences in vascular density (Figure 4A). In prefrontal cortex gray matter, it was found to be comparable between depressed suicides and controls $[6.9 \pm 0.6 \%$ vs. $7.4 \pm 0.5 \%$ coverage; $p$ (uncorrected) $=0.55$, $p($ corrected $)=0.76$; Figures 4B-E]. Similar results were found in the mediodorsal thalamus and caudate nucleus, in which vascular densities were also comparable between depressed suicides and controls [thalamus: $7.2 \pm 0.4 \%$ vs. $7.5 \pm 0.4 \%$ coverage; $p$ (uncorrected) $=0.58, p$ (corrected) $=0.96$; caudate: $8.1 \pm 0.3 \%$ vs. $7.7 \pm 0.5 \%$ coverage; $p$ $($ uncorrected $)=0.46, p($ corrected $)=0.28]$. Only in prefrontal cortex white matter was there a significant group difference in vascular density, with greater vascularization in depressed suicides than in controls $[3.4 \pm 1.2 \%$ vs. $2.6 \pm 0.2 \%$ coverage; $p($ uncorrected $)=0.003, p($ corrected $)=0.002]$.

\section{DISCUSSION}

To our knowledge, this is the first study investigating VIM-IR astrocytes in MDD and suicide, and the first cross-regional study of astrocyte density or morphology in MDD. This is one of few post-mortem studies comparing the same samples with more than one astrocytic marker. The main results (1) further support previous reports of greater differences in GFAP expression in subcortical regions than in cortical regions of depressed suicides (20); (2) show that VIM-IR astrocyte densities are even more robustly decreased (without morphological changes) in cortical regions; (3) reveal altered CD31-IR vascular density in the PFC WM of depressed suicides. Presenting our data as densities using a stereological approach avoided the potential bias in counting from cortical volumes altered by depression or other factors. As depression generally decreases cortical volumes (38), a non-stereological approach would likely give rise to increased or unchanged cortical astrocyte densities in case samples. However, previous stereological post-mortem studies have reported decreased glial densities in the prefrontal cortex in depression $(10,39)$, where we now report a decreased density of VIM-IR astrocytes.

\section{Astrocyte Densities}

In this study, we revealed lower GFAP-IR and VIM-IR astrocyte densities in both cortical and subcortical brain samples from well-characterized depressed suicides compared to matched controls (Figure 5). For GFAP-IR astrocytes, this decrease was statistically significant in all regions except for the prefrontal cortex white matter $(p=0.053)$. This reveals a widespread alteration in GFAP-IR astrocytes throughout a number of cerebral networks in depressed individuals. The density of VIM-IR astrocytes was also assessed in the same subjects and regions. As our previous study showed that VIM mostly labels a subset of GFAP-IR astrocytes (8), this approach helped clarify whether reduced GFAP-IR astrocyte densities in depressed suicides reflects a reduction in astrocyte density as opposed to a reduction of GFAP immunoreactivity. In depressed suicides, the density of VIM-IR astrocytes was strongly and significantly lower in the prefrontal cortex and caudate nucleus, but not in the mediodorsal thalamus, in which exceedingly rare VIM-IR astrocytes are observed in controls. The lower densities of both GFAP-IR and VIM-IR astrocytes favors a hypothesis of a reduced number of astrocytes over a reduced reactive profile of astrocytes in depressed suicides.

The brain regions examined in this study are closely associated in a prominent frontal-subcortical circuit implicated in executive function-neurons project from the caudate nucleus to the mediodorsal thalamus and then to the dorsomedial prefrontal cortex gray matter (40). This executive circuit has been found to be dysregulated in depression, with patients displaying a relatively lower resting state connectivity between the prefrontal cortex and either the striatum or the thalamus are more likely to have a positive treatment response to repetitive transcranial magnetic stimulation of the prefrontal cortex (41). The connectivity of the prefrontal cortex seems essential to the neuroanatomy of depression-no single brain region consistently associates with MDD when lesioned, however, brain regions with relatively high resting state connectivity to the dorsomedial prefrontal cortex are consistently associated with MDD following lesions (42). In the context of our results, astrocyte dysfunction may directly regulate resting state functional connectivity to the prefrontal cortex, as astrocytes mediate vasodilation responsible for changes in regional cerebral blood flow. If our results do represent a reduction in numbers of astrocytes, this may represent a compensatory mechanism by which the brain is indirectly reducing functional connectivity by impairing the efficiency of gliovascular coupling in a circuit that becomes hyperconnected in depression.

A reduction in GFAP-IR astrocyte density in multiple brain regions from depressed suicides supports a strong consensus in the literature for decreased regional GFAP protein levels in post-mortem studies and animal models of depression (21). Our findings also support post-mortem studies on depression which have found semi-qualitative decreases in the number of GFAP-IR astrocytes in other brain regions, such as the hippocampus (43). We observed greater differences in GFAP-IR astrocyte densities in subcortical regions than in cortical regions, which is in agreement with a previous report of GFAP protein levels being even more downregulated in the mediodorsal thalamus and caudate nucleus than in cortical regions of depressed suicides relative to controls (20). This previous study also found the mediodorsal thalamus and caudate nucleus have greater regional GFAP expression levels than many cortical regions in healthy adults, and so our findings indicate that brain regions that normally have a high GFAP expression in healthy adults may have particularly strong depression-related changes in GFAP-IR astrocyte densities. The association between regional 


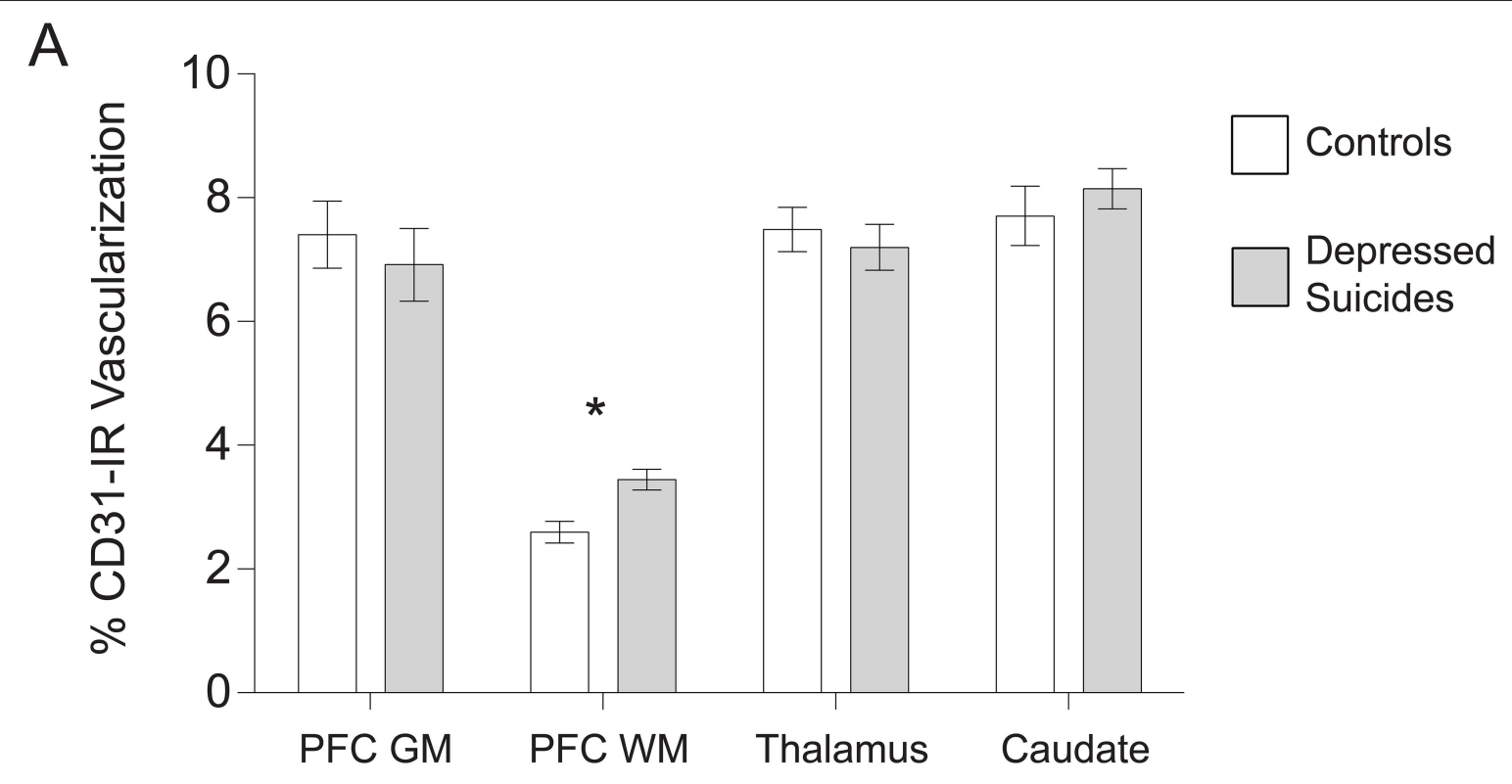

\section{Controls}
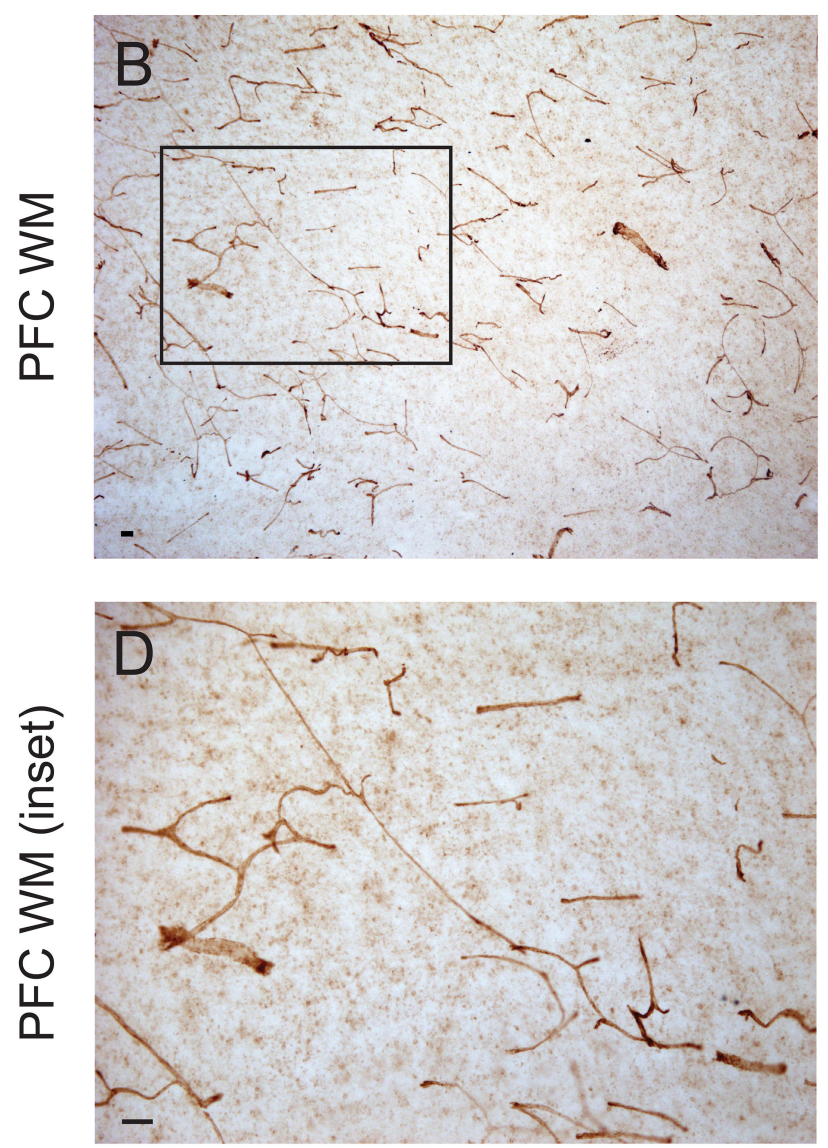

Depressed Suicides
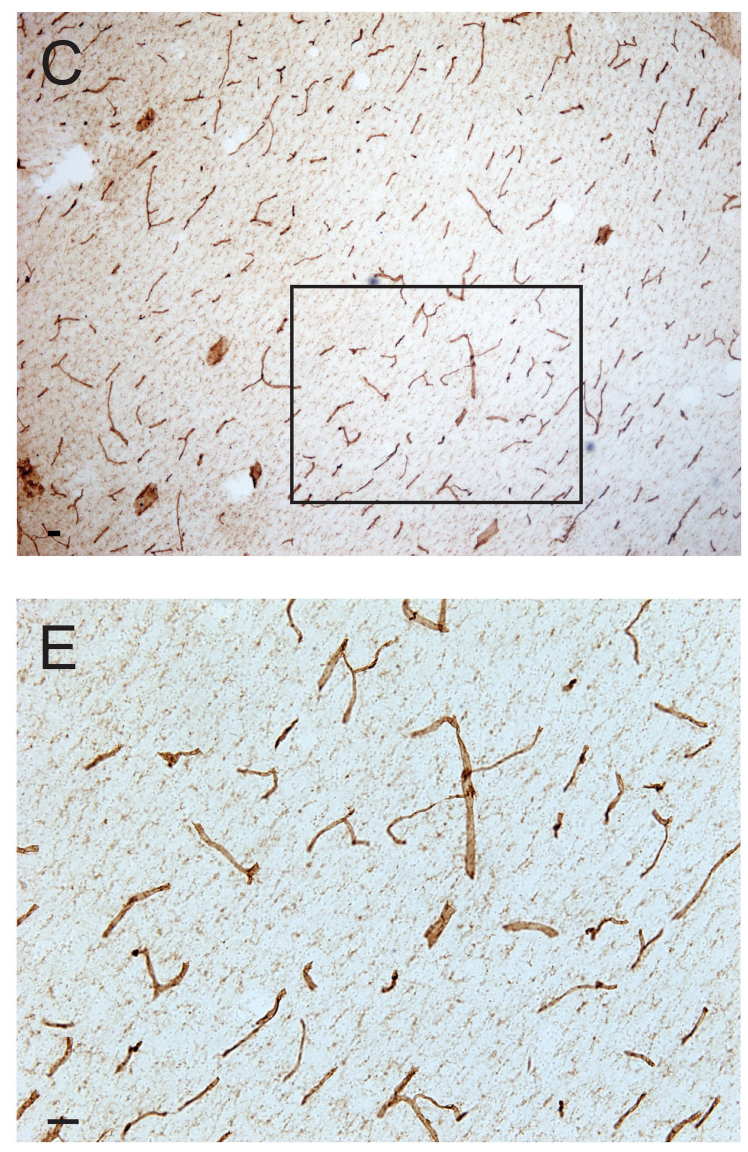

FIGURE 4 | Increased CD31-IR vascular density in cortical white matter from depressed suicides relative to controls. (A) Representative micrographs showing that CD31-IR vascular density in the prefrontal cortex white matter (PFC WM). Scale bars $=50 \mu \mathrm{m}$. (B-E) Depressed suicides had a significantly higher vascular density than controls in the mediodorsal thalamus. No group differences were observed in the prefrontal cortex gray matter (PFC GM), the caudate nucleus or the mediodorsal thalamus. ${ }^{*} p \leq 0.05 ; n=10$; unpaired $t$-tests corrected for age, $\mathrm{pH}$ and post-mortem interval. 


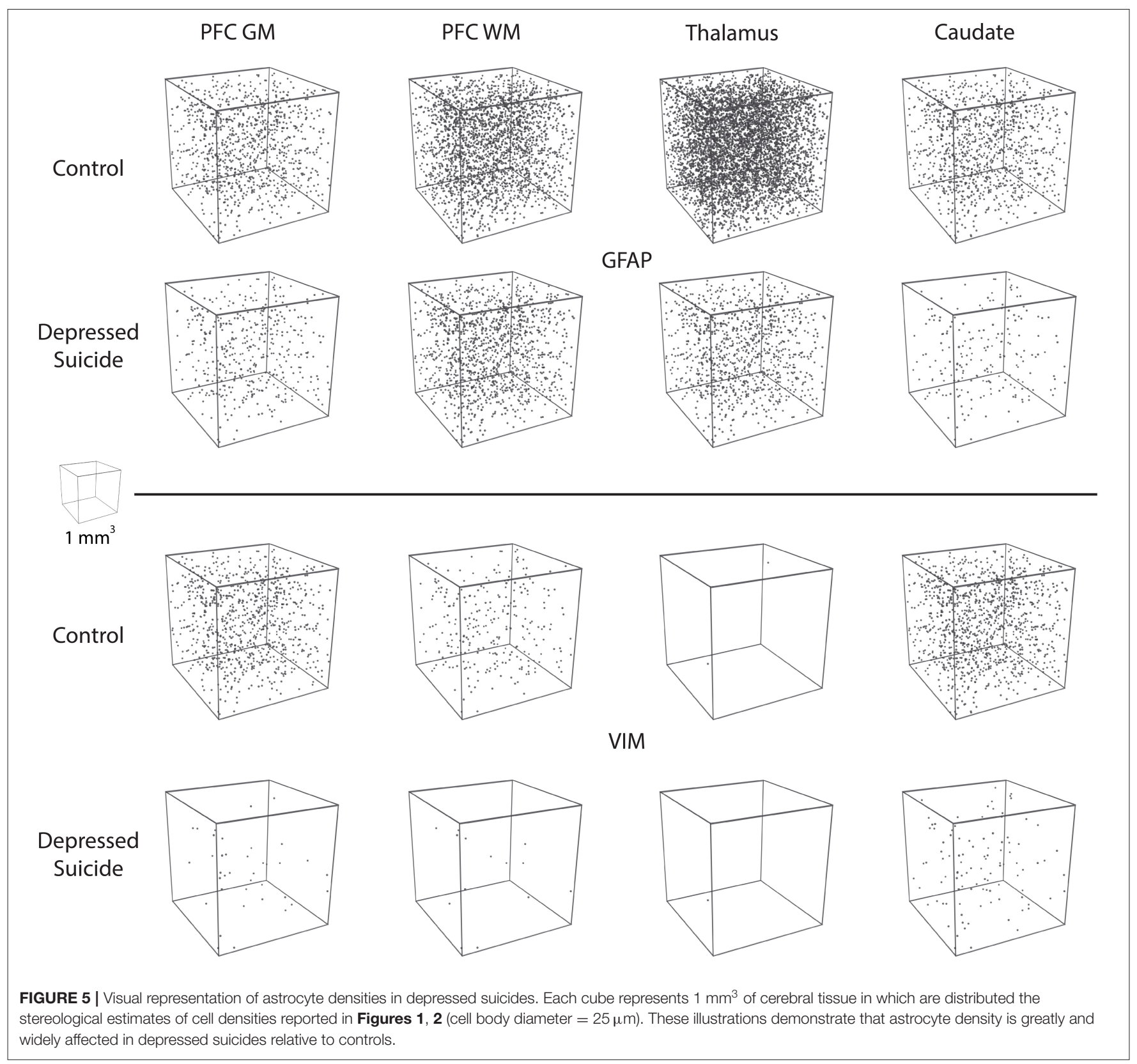

GFAP expression and GFAP-IR astrocyte density is supported by a previous study that correlated low regional GFAP protein levels with low GFAP-IR area fraction in the dorsomedial prefrontal cortex gray matter of depressed suicides (14), which may well resemble a reduction in cell density given our findings. Although we found no significant difference in GFAP-IR astrocyte density in dorsomedial prefrontal cortex white matter, a previous study reported a significantly lower density of GFAP-IR astrocytes in the ventromedial prefrontal cortex white matter of depressed suicides-a difference that was 5-fold greater than was observed for dorsomedial prefrontal cortex white matter in the present study (17). This suggests that prefrontal cortex white matter may have a particularly strong subregional heterogeneity for depression-related changes in GFAP-IR astrocyte density.

While VIM-IR astrocytes clearly represent a minority of the total astrocyte population in healthy controls (8), VIM is a valuable marker as it is strongly immunoreactive in both cell body and processes. The group differences in astrocyte density in the prefrontal cortex gray matter and caudate nucleus were twice as great for VIM-IR astrocytes than for GFAP-IR astrocytes, indicating astrocytes strongly expressing VIM may be especially affected in depressed suicides. The lack of a significant decrease of VIM-IR astrocyte density in the mediodorsal thalamus was unsurprising, given that many sections from the thalamus of control subjects in this and our previous study contained no 
VIM-IR astrocytes at all (8). While the density of VIM-IR astrocytes is extremely low in our analyses of the mediodorsal thalamus, VIM-IR astrocytes have previously been reported to be almost absent in post-mortem hippocampal samples (44). This paucity of VIM-IR astrocytes in certain brain regions is further suggested by the substantial differences in astrocyte density in different nuclei of the thalamus in mouse brain (45). However, we suspect this indicates an unrecognized functional role of thalamic astrocytes that does not require VIM expression. As VIM expression affects the speed of vesicular transport and the expression of other astrocytic intermediate filaments, this low VIM expression may reflect both structural and functional differences for thalamic astrocytes that otherwise express GFAP $(46,47)$. Following from this, the widespread loss of VIMIR astrocytes in MDD suggests the loss vesicular trafficking and cell division properties more commonly seen in non-VIMIR astrocytes.

To date, only one other post-mortem study has assessed VIM in the context of depression and found no significant decrease of VIM mRNA levels in MDD in the anterior cingulate cortex of depressed individuals who died by natural causes (not by suicide) (48). Although this may reflect an effect unique to the anterior cingulate cortex, this does not reliably imply no change of VIM-IR astrocyte density in the ACC, given that the most VIM expression in the brain is found in vascular endothelial cells $(8,49)$. As for astrocytes labeled with glutamine synthetase, there is a reduced number of VIM-IR astrocytes in post-mortem brain samples from individuals with MDD, but due to its expression in other cell types, this effect is not detected at the regional level of protein or mRNA (50). The reduction of VIMIR astrocyte density in depressed suicides suggests that reduced GFAP levels do not correspond specifically to GFAP dysfunction, but rather a widespread loss or dysfunction of astrocytes. A panastrocytic marker like Aldh1L1 will be needed to infer whether only reactive astrocytes expressing VIM and GFAP are affected in depression; however, this seems unlikely, as genes specific to both non-reactive and reactive astrocytes are downregulated in many brain regions in depressed suicides $(27,28,51)$ and depressed non-suicides (52). We do not exclude the possibility that astrocyte density differences may differ in post-mortem samples from depressed non-suicides. However, investigations having compared regional astrocyte densities between depressed individuals having died by suicide or non-suicidal causes found no significant difference between groups $(16,53)$. As our depressed suicide cohort died by hanging, one might expect vascular changes from asphyxia may have systematically altered gliovascular interactions in a way that might not be observed for other methods of suicide.

\section{Vascular Densities}

In a previous study, our group showed that regional astrocyte density inversely correlates with regional vascular density in post-mortem brain samples from controls (8). In the present study, brain regions from depressed suicides had significantly lower astrocyte density than controls, and so we anticipated that they may also have a correspondingly greater vascular density. Depressed suicides had a significantly different (higher) vascular density than controls only in prefrontal cortex white matter-no other significant differences or general trends were observed for regional vascular density. This finding suggests that gliovascular interactions in cortical white matter may be preferentially affected by depression, as also suggested by a previous report of reduced coverage of blood vessels by astrocytic endfeet in prefrontal cortex gray matter, but not white matter (26). It is also likely that vascular density does not reflect the full extent of vascular changes in depressed individuals, such as the known reduction in claudin-5 protein levels in post-mortem vascular endothelial cells of the nucleus accumbens in depressed suicides, which in animal models has been associated with an abnormally increased permeability of the blood brain barrier (54, 55). Future studies on gliovascular interactions in depression may need to resolve this with finer precision with a non-stereological method, given the absence of a clear region-wide relationship between astrocyte and vascular density in depression.

\section{Limitations and Future Directions}

The two main limitations of this study were its relatively small sample size and lack of samples from females. Female samples were not included due to the low availability of such samples from depressed suicides which could be effectively matched for factors that are known to greatly affect astrocyte regional densities and gene expression in post-mortem samples, including age and post-mortem interval $(12,27)$. Furthermore, some important questions remain that could not be assessed with the current sample, including whether the number, duration and severity of depressive episodes may have an incidence on VIM-IR or GFAP-IR astrocyte densities. In conclusion, different brain regions in depressed suicides exhibit robust reductions in VIM-IR astrocyte densities that are even greater than those for GFAP-IR astrocytes. Our data also more generally revealed a consistent cross-regional trend for reduced astrocyte densities in depressed suicides, and a unique change in vascular density in the prefrontal cortex white matter. With the minor exception of fibrous astrocytes in the prefrontal cortex, there were no clear changes in the morphology of VIM-IR astrocytes that we could most clearly and precisely observe, indicating that depression has a larger and more widespread effect on astrocyte density than on astrocyte morphology in mood-associated brain regions. In the future, single cell sequencing approaches will be informative for establishing whether the function of these smaller populations of cells in depressed suicides are altered in depressed suicides.

\section{DATA AVAILABILITY STATEMENT}

The raw data supporting the conclusions of this article will be made available by the authors, without undue reservation.

\section{ETHICS STATEMENT}

The studies involving human participants were reviewed and approved by Douglas Hospital Research Ethics Board of the Douglas Mental Health University Institute. The patients/participants provided their written informed consent to participate in this study. 


\section{AUTHOR CONTRIBUTIONS}

LO'L and NM conceived the project, designed the experiments, and drafted the manuscript. $\mathrm{CB}, \mathrm{MD}$, and LO'L conducted tissue processing. LO'L conducted the IHC experiments, performed vascular density and astrocyte morphometry experiments, and analyzed all the data. LO'L and JM performed the cell counting experiments. All authors contributed to the interpretation of the results in addition to participating in the finalization of the manuscript.

\section{FUNDING}

This study was funded by an NSERC Discovery Grant and a CIHR Project Grant to NM. The Molecular and

\section{REFERENCES}

1. Andriezen WL. The neuroglia elements in the human brain. Br Med J. (1893) 2:227-30. doi: 10.1136/bmj.2.1700.227

2. Norenberg MD, Martinez-Hernandez A. Fine structural localization of glutamine synthetase in astrocytes of rat brain. Brain Res. (1979) 161:303-10. doi: 10.1016/0006-8993(79)90071-4

3. Rothstein JD, Dykes-Hoberg M, Pardo CA, Bristol LA, Jin L, Kuncl RW, et al. Knockout of glutamate transporters reveals a major role for astroglial transport in excitotoxicity and clearance of glutamate. Neuron. (1996) 16:67586. doi: 10.1016/S0896-6273(00)80086-0

4. Duan S, Anderson CM, Stein BA, Swanson RA. Glutamate induces rapid upregulation of astrocyte glutamate transport and cellsurface expression of GLAST. J Neurosci. (1999) 19:10193-200. doi: 10.1523/JNEUROSCI.19-23-10193.1999

5. Cui Y, Yang Y, Ni Z, Dong Y, Cai G, Foncelle A, et al. Astroglial Kir4.1 in the lateral habenula drives neuronal bursts in depression. Nature. (2018) 554:323-7. doi: 10.1038/nature25752

6. Covelo A, Araque A. Neuronal activity determines distinct gliotransmitter release from a single astrocyte. eLife. (2018) 7:e32237. doi: 10.7554/eLife.32237.018

7. Oberheim NA, Takano T, Han X, He W, Lin JHC, Wang F, et al. Uniquely hominid features of adult human astrocytes. J Neurosci. (2009) 29:3276-87. doi: 10.1523/JNEUROSCI.4707-08.2009

8. O'Leary LA, Davoli MA, Belliveau C, Tanti A, Ma C, Farmer WT, et al. Characterization of vimentin-immunoreactive astrocytes in the human brain. Front Neuroanat. (2020) 14:31. doi: 10.3389/fnana.2020.00031

9. Öngür D, Drevets WC, Price JL. Glial reduction in the subgenual prefrontal cortex in mood disorders. PNAS. (1998) 95:13290-5. doi: $10.1073 /$ pnas.95.22.13290

10. Rajkowska G, Miguel-Hidalgo JJ, Wei J, Dilley G, Pittman SD, Meltzer HY, et al. Morphometric evidence for neuronal and glial prefrontal cell pathology in major depression**see accompanying editorial, in this issue. Biol Psychiatry. (1999) 45:1085-98. doi: 10.1016/S0006-3223(99)00041-4

11. Bowley MP, Drevets WC, Öngür D, Price JL. Low glial numbers in the amygdala in major depressive disorder. Biol Psychiatry. (2002) 52:404-12. doi: 10.1016/S0006-3223(02)01404-X

12. Miguel-Hidalgo JJ, Baucom C, Dilley G, Overholser JC, Meltzer HY, Stockmeier CA, et al. Glial fibrillary acidic protein immunoreactivity in the prefrontal cortex distinguishes younger from older adults in major depressive disorder. Biol Psychiatry. (2000) 48:861-73. doi: 10.1016/S0006-3223(00)00999-9

13. Webster MJ, Knable MB, Johnston-Wilson N, Nagata K, Inagaki M, Yolken $\mathrm{RH}$. Immunohistochemical localization of phosphorylated glial fibrillary acidic protein in the prefrontal cortex and hippocampus from patients with schizophrenia, bipolar disorder, and depression. Brain Behav Immun. (2001) 15:388-400. doi: 10.1006/brbi.2001.0646
Cellular Microscopy Platform and the Douglas-Bell Canada Brain Bank (DBCBB) are partly funded by a Healthy Brains for Healthy Lives (CFREF) Platform Grant to GT and NM. The DBCBB is also funded by the Réseau Québécois sur le suicide, le troubles de l'humeur et les troubles associés (FRQS).

\section{ACKNOWLEDGMENTS}

The present study used the services of the Douglas-Bell Canada Brain Bank and of the Molecular and Cellular Microscopy Platform at the Douglas Hospital Research Center. The authors are grateful to Maâmar Bouchouka, Josée Prud'homme, Dominique Mirault, and Melina Jaramillo Garcia for their kind assistance.

14. Si X, Miguel-Hidalgo JJ, O’Dwyer G, Stockmeier CA, Rajkowska G. Agedependent reductions in the level of glial fibrillary acidic protein in the prefrontal cortex in major depression. Neuropsychopharmacology. (2004) 29:2088-96. doi: 10.1038/sj.npp.1300525

15. Altshuler LL, Abulseoud OA, Foland-Ross L, Bartzokis G, Chang S, Mintz J, et al. Amygdala astrocyte reduction in subjects with major depressive disorder but not bipolar disorder. Bipolar Disord. (2010) 12:541-9. doi: 10.1111/j.1399-5618.2010.00838.x

16. Cobb JA, O’Neill K, Milner J, Mahajan GJ, Lawrence TJ, May WL, et al. Density of GFAP-immunoreactive astrocytes is decreased in left hippocampi in major depressive disorder. Neuroscience. (2016) 316:209-20. doi: 10.1016/j.neuroscience.2015.12.044

17. Rajkowska G, Legutko B, Moulana M, Syed M, Romero DG, Stockmeier CA, et al. Astrocyte pathology in the ventral prefrontal white matter in depression. J Psychiatr Res. (2018) 102:150-8. doi: 10.1016/j.jpsychires.2018.04.005

18. Chandley MJ, Szebeni K, Szebeni A, Crawford J, Stockmeier CA, Turecki $\mathrm{G}$, et al. Gene expression deficits in pontine locus coeruleus astrocytes in men with major depressive disorder. J Psychiatry Neurosci. (2013) 38:276-84. doi: $10.1503 /$ jpn. 120110

19. Fatemi SH, Laurence JA, Araghi-Niknam M, Stary JM, Schulz SC, Lee S, et al. Glial fibrillary acidic protein is reduced in cerebellum of subjects with major depression, but not schizophrenia. Schizophr Res. (2004) 69:317-23. doi: 10.1016/j.schres.2003.08.014

20. Torres-Platas SG, Nagy C, Wakid M, Turecki G, Mechawar N. Glial fibrillary acidic protein is differentially expressed across cortical and subcortical regions in healthy brains and downregulated in the thalamus and caudate nucleus of depressed suicides. Mol Psychiatry. (2016) 21:509-15. doi: $10.1038 / \mathrm{mp} .2015 .65$

21. Kim R, Healey KL, Sepulveda-Orengo MT, Reissner KJ. Astroglial correlates of neuropsychiatric disease: from astrocytopathy to astrogliosis. Prog Neuro Psychopharmacol Biol Psychiatry. (2018) 87:126-46. doi: 10.1016/j.pnpbp.2017.10.002

22. Sofroniew MV, Vinters HV. Astrocytes: biology and pathology. Acta Neuropathol. (2010) 119:7-35. doi: 10.1007/s00401-009-0619-8

23. Gos T, Schroeter ML, Lessel W, Bernstein H-G, Dobrowolny H, et al. S100Bimmunopositive astrocytes and oligodendrocytes in the hippocampus are differentially afflicted in unipolar and bipolar depression: a postmortem study. J Psychiatric Res. (2013) 47:1694-9. doi: 10.1016/j.jpsychires.2013.07.005

24. Hamidi M, Drevets WC, Price JL. Glial reduction in amygdala in major depressive disorder is due to oligodendrocytes. Biol Psychiatry. (2004) 55:5639. doi: 10.1016/j.biopsych.2003.11.006

25. Torres-Platas SG, Hercher C, Davoli MA, Maussion G, Labonté B, Turecki G, et al. Astrocytic hypertrophy in anterior cingulate white matter of depressed suicides. Neuropsychopharmacology. (2011) 36:2650-8. doi: $10.1038 /$ npp.2011.154

26. Rajkowska G, Hughes J, Stockmeier CA, Javier Miguel-Hidalgo J, Maciag D. Coverage of blood vessels by astrocytic endfeet is reduced 
in major depressive disorder. Biol Psychiatry. (2013) 73:613-21. doi: 10.1016/j.biopsych.2012.09.024

27. Nagy C, Suderman M, Yang J, Szyf M, Mechawar N, Ernst C, et al. Astrocytic abnormalities and global DNA methylation patterns in depression and suicide. Mol Psychiatry. (2015) 20:320-8. doi: 10.1038/mp.2014.21

28. Zhao J, Verwer RWH, van Wamelen DJ, Qi X-R, Gao S-F, Lucassen PJ, et al. Prefrontal changes in the glutamate-glutamine cycle and neuronal/glial glutamate transporters in depression with and without suicide. J Psychiatr Res. (2016) 82:8-15. doi: 10.1016/j.jpsychires.2016.06.017

29. Ernst C, Nagy C, Kim S, Yang JP, Deng X, Hellstrom IC, et al. Dysfunction of astrocyte connexins 30 and 43 in dorsal lateral prefrontal cortex of suicide completers. Biol Psychiatry. (2011) 70:312-9. doi: 10.1016/j.biopsych.2011.03.038

30. Miguel-Hidalgo JJ, Wilson BA, Hussain S, Meshram A, Rajkowska G, Stockmeier CA. Reduced connexin 43 immunolabeling in the orbitofrontal cortex in alcohol dependence and depression. J Psychiatr Res. (2014) 55:101-9. doi: 10.1016/j.jpsychires.2014.04.007

31. Tanti A, Lutz P-E, Kim J, O'Leary L, Théroux J-F, Turecki G, et al. Evidence of decreased gap junction coupling between astrocytes and oligodendrocytes in the anterior cingulate cortex of depressed suicides. Neuropsychopharmacology. (2019) 44:2099-111. doi: 10.1038/s41386-019-0471-z

32. Dahl D. The vimentin-GFA protein transition in rat neuroglia cytoskeleton occurs at the time of myelination. J Neurosci Res. (1981) 6:741-8. doi: 10.1002/jnr.490060608

33. Tomokane N, Iwaki T, Tateishi J, Iwaki A, Goldman JE. Rosenthal fibers share epitopes with alpha B-crystallin, glial fibrillary acidic protein, and ubiquitin, but not with vimentin. Immunoelectron microscopy with colloidal gold. Am J Pathol. (1991) 138:875-85.

34. Wilhelmsson U, Li L, Pekna M, Berthold C-H, Blom S, et al. Absence of glial fibrillary acidic protein and vimentin prevents hypertrophy of astrocytic processes and improves post-traumatic regeneration. J Neurosci. (2004) 24:5016-21. doi: 10.1523/JNEUROSCI.0820-04.2004

35. Yamada T, Kawamata T, Walker DG, McGeer PL. Vimentin immunoreactivity in normal and pathological human brain tissue. Acta Neuropathologica. (1992) 84:157-62. doi: 10.1007/BF00311389

36. Abdellah M, Hernando J, Eilemann S, Lapere S, Antille N, Markram H, et al. NeuroMorphoVis: a collaborative framework for analysis and visualization of neuronal morphology skeletons reconstructed from microscopy stacks. Bioinformatics. (2018) 34:i574-82. doi: 10.1093/bioinformatics/bty231

37. Motulsky HJ, Brown RE. Detecting outliers when fitting data with nonlinear regression-a new method based on robust nonlinear regression and the false discovery rate. BMC Bioinformatics. (2006) 7:123. doi: 10.1186/1471-2105-7-123

38. Grieve SM, Korgaonkar MS, Koslow SH, Gordon E, Williams LM. Widespread reductions in gray matter volume in depression. NeuroImage Clin. (2013) 3:332-9. doi: 10.1016/j.nicl.2013.08.016

39. Cotter D, Mackay D, Chana G, Beasley C, Landau S, Everall IP. Reduced neuronal size and glial cell density in area 9 of the dorsolateral prefrontal cortex in subjects with major depressive disorder. Cerebral Cortex. (2002) 12:386-94. doi: 10.1093/cercor/12.4.386

40. Bonelli RM, Cummings JL. Frontal-subcortical circuitry and behavior. Dialogues Clin Neurosci. (2007) 9:141-51. doi: 10.31887/DCNS.2007.9.2/rbonelli

41. Salomons TV, Dunlop K, Kennedy SH, Flint A, Geraci J, Giacobbe $\mathrm{P}$, et al. Resting-state cortico-thalamic-striatal connectivity predicts response to dorsomedial prefrontal rTMS in major depressive disorder. Neuropsychopharmacology. (2014) 39:488-98. doi: 10.1038/npp. 2013.222

42. Padmanabhan JL, Cooke D, Joutsa J, Siddiqi SH, Ferguson M, Darby RR, et al. A human depression circuit derived from focal brain lesions. Biol Psychiatry. (2019) 86:749-58. doi: 10.1016/j.biopsych.2019.07.023
43. Müller MB, Lucassen PJ, Yassouridis A, Hoogendijk WJG, Holsboer F, Swaab DF. Neither major depression nor glucocorticoid treatment affects the cellular integrity of the human hippocampus. Eur J Neurosci. (2001) 14:1603-12. doi: 10.1046/j.0953-816x.2001.01784.x

44. Arnold SE, Franz BR, Trojanowski JQ, Moberg PJ, Gur RE. Glial fibrillary acidic protein-immunoreactive astrocytosis in elderly patients with schizophrenia and dementia. Acta Neuropathologica. (1996) 91:269-77. doi: 10.1007/s004010050425

45. Emsley JG, Macklis JD. Astroglial heterogeneity closely reflects the neuronaldefined anatomy of the adult murine CNS. Neuron Glia Biol. (2006) 2:175-86. doi: 10.1017/S1740925X06000202

46. Jing R, Wilhelmsson U, Goodwill W, Li L, Pan Y, Pekny M, et al. Synemin is expressed in reactive astrocytes in neurotrauma and interacts differentially with vimentin and GFAP intermediate filament networks. J Cell Sci. (2007) 120:1267-1277. doi: 10.1242/jcs.03423

47. Vardjan N, Gabrijel M, Potokar M, Švajger U, Kreft M, Jeras M, et al. IFN- $\gamma$-induced increase in the mobility of MHC class II compartments in astrocytes depends on intermediate filaments. J Neuroinflammation. (2012) 9:144. doi: 10.1186/1742-2094-9-144

48. Qi X-R, Kamphuis W, Shan L. Astrocyte changes in the prefrontal cortex from aged non-suicidal depressed patients. Front Cell Neurosci. (2019) 13:503. doi: $10.3389 /$ fncel.2019.00503

49. Nagy C, Maitra M, Tanti A, Suderman M, Théroux J-F, Davoli MA, et al. Single-nucleus transcriptomics of the prefrontal cortex in major depressive disorder implicates oligodendrocyte precursor cells and excitatory neurons. Nat Neurosci. (2020) 23:771-81. doi: 10.1038/s41593-020-0621-y

50. Bernstein H-G, Meyer-Lotz G, Dobrowolny H, Bannier J, Steiner J, Walter M, et al. Reduced density of glutamine synthetase immunoreactive astrocytes in different cortical areas in major depression but not in bipolar I disorder. Front Cell Neurosci. (2015) 9:273. doi: 10.3389/fncel.2015.00273

51. Nagy C, Torres-Platas SG, Mechawar N, Turecki G. Repression of astrocytic connexins in cortical and subcortical brain regions and prefrontal enrichment of H3K9me3 in depression and suicide. Int J Neuropsychopharmacol. (2017) 20:50-7. doi: 10.1093/ijnp/pyw071

52. Zhao J, Verwer RWH, Gao S-F, Qi X-R, Lucassen PJ, Kessels HW, et al. Prefrontal alterations in GABAergic and glutamatergic gene expression in relation to depression and suicide. J Psychiatr Res. (2018) 102:261-74. doi: 10.1016/j.jpsychires.2018.04.020

53. Zhao J, Lucassen PJ, Swaab DF. Suicide is a confounder in postmortem studies on depression. Biol Psychiatry. (2019) 86:e37-40. doi: 10.1016/j.biopsych.2019.04.015

54. Menard C, Pfau ML, Hodes GE, Kana V, Wang VX, Bouchard $\mathrm{S}$, et al. Social stress induces neurovascular pathology promoting depression. Nat Neurosci. (2017) 20:1752-60. doi: 10.1038/s41593-0170010-3

55. Dudek KA, Dion-Albert L, Lebel M, LeClair K, Labrecque S, Tuck E, et al. Molecular adaptations of the blood-brain barrier promote stress resilience vs. depression. PNAS. (2020) 117:3326-36. doi: 10.1073/pnas.1914 655117

Conflict of Interest: The authors declare that the research was conducted in the absence of any commercial or financial relationships that could be construed as a potential conflict of interest.

Copyright (๑) 2021 O'Leary, Belliveau, Davoli, Ma, Tanti, Turecki and Mechawar. This is an open-access article distributed under the terms of the Creative Commons Attribution License (CC BY). The use, distribution or reproduction in other forums is permitted, provided the original author(s) and the copyright owner(s) are credited and that the original publication in this journal is cited, in accordance with accepted academic practice. No use, distribution or reproduction is permitted which does not comply with these terms. 